\title{
Zoning the territory of the Republic of Kazakhstan as to the risk of rabies among various categories of animals
}

\author{
Sarsenbay K. Abdrakhmanov, ${ }^{1}$ Akhmetzhan A. Sultanov, ${ }^{2}$ Kanatzhan K. Beisembayev, \\ Fedor I. Korennoy, ${ }^{3}$ Dosym B. Kushubaev, ${ }^{1}$ Ablaikhan S. Kadyrov' ${ }^{1}$ \\ 'S. Seifullin Kazakh Agro-Technical University, Astana; ${ }^{2}$ Kazakh Research Veterinary \\ Institute, Almaty, Kazakhstan; ${ }^{3}$ Federal Center for Animal Health (FGBI ARRIAH), \\ Vladimir, Russia
}

\section{Abstract}

This paper presents the zoning of the territory of the Republic of Kazakhstan with respect to the risk of rabies outbreaks in domestic and wild animals considering environmental and climatic conditions. The national database of rabies outbreaks in Kazakhstan in the period 2003-2014 has been accessed in order to find which zones are consistently most exposed to the risk of rabies in animals. The database contains information on the cases in demes of farm livestock, domestic animals and wild animals. To identify the areas with the highest risk of outbreaks, we applied the maximum entropy modelling method. Designated outbreaks were used as input presence data, while the bioclim set of ecological and climatic variables, together with some geographic factors, were used as explanatory variables. The model demonstrated a high predictive ability. The area under the curve for farm livestock was 0.782 , for domestic animals -0.859 and for wild animals 0.809 . Based on the model, the map of integral risk was designed by following four categories: negligible risk (disease-free or favourable zone), low risk (surveillance zone), medium risk (vaccination zone), and high risk (unfavourable zone). The map was produced to allow developing a set of preventive measures and is expected to contribute to a better distribution of supervisory efforts from the veterinary service of the country.

Correspondence: Sarsenbay K. Abdrakhmanov, S. Seifullin Kazakh AgroTechnical University, 62 av. Pobeda, 010011 Astana, Kazakhstan.

Tel. +77.013.881467.

E-mail: S_abdrakhmanov@mail.ru

Key words: Kazakhstan; GIS; MaxEnt; Rabies; Zoning.

Received for publication: 21 November 2015.

Revision received: 19 January 2016.

Accepted for publication: 20 January 2016.

(C) Copyright S.K. Abdrakhmanov et al., 2016

Licensee PAGEPress, Italy

Geospatial Health 2016; 11:429

doi:10.4081/gh.2016.429

This article is distributed under the terms of the Creative Commons Attribution Noncommercial License (CC BY-NC 4.0) which permits any noncommercial use, distribution, and reproduction in any medium, provided the original author(s) and source are credited.

\section{Introduction}

According to the World Health Organization, rabies ranks fifth among all infectious diseases based on the economic loss suffered from the disease. The disease, reported in 113 countries to date, is characterised by an acute course with overt signs of polyencephalomyelitis. Mortality is $100 \%$ in the absence of immediate treatment. More than 55,000 people and more than one million animals in the world die annually due to this infection. Direct damage caused by rabies costs society about 4 billion EUR per year (Nouvellet et al., 2013; Robardet et al., 2013).

Rabies is one of especially dangerous zoonotic diseases with uneven spread of infection that is sharply delineated in the world. It is registered in every continent except Australia and Antarctica (Zavodskih and Sludov, 2007; Makarov et al., 2008; Smreczak et al., 2009, 2012; Orlowska et al., 2011; Youla et al., 2014). The same pattern of growth of rabies in the world has also been observed in the Republic of Kazakhstan (RK). Curently, the number of rabies cases recorded in animals (fox, raccoon dogs, wolves, cats and cattle) tends to rise by an average of $7 \%$ annually. About 700 heads of farm livestock (more than $50 \%$ of which refers to cattle and up to $25 \%$ to small ungulates) die of rabies every year in the (Abdrakhmanov et al., 2010). Thus, the rabies epizootic situation has become extremely difficult in most regions of the country. Natural foci of infection have sharply intensified with the number of cases among different species increasing, including human cases with fatal outcome (Bersagurov, 2002; Zholshorinov and Sansyzbayev, 2004). Current measures held in RK to control rabies mainly include: i) oral vaccination of wild animals within outbreaks and adjacent areas; ii) forced and preventive vaccination of susceptible livestock and domestic carnivores. The vaccination of the latter is needed as a necessary measure of urban control aiming to limit spread of the disease to humans. In addition, strict account and control of stray and domestic carnivores as well as awareness-raising activities among the human population are part of the main control activities enforced. Despite these ongoing efforts, it is still not possible to control the disease and prevent its spread. This fact is due to the factors mentioned, in particular the presence of natural foci of infection (Domsky, 2002; Chubirko et al., 2003; Dudnikov, 2003).

The disease is constantly under scrupulous attention of the veterinary services in RK, which plans surveillance campaigns and decides which demes of livestock and wild animals to subject to mass vaccination. One of traditional activities undertaken by the veterinary service is zoning of the country in accordance with the presence of rabies outbreaks in the past and the probability of their occurrence in the future. Generally, four zones are distinguished: i) unfavourable zones, where outbreaks are presently recorded; ii) vaccination zones, where outbreaks have not been recorded for three years and where vaccination of susceptible livestock is being carried out; iii) surveillance zones, 
which are areas directly adjacent to the vaccination zones; and iv) favourable zones, where outbreaks of the disease have not been recorded.

Zoning is only carried out with regard to existing outbreaks and does not involve research. The purpose of zoning is to allocate veterinary service resources for surveillance and vaccination to prevent the development of epizootic rabies and its diffusion into zones designated as favourable.

The objective of the present study was to institute modern analytical methods for zoning that consider not only the presence or absence of outbreaks on a given territory, but also the probability of their occurrence in the future based on the aggregation of ecological and geographical characteristics on the territory in question.

\section{Materials and Methods}

\section{Study area}

RK, covering an area of $2,724,902 \mathrm{~km}^{2}$, is the $9^{\text {th }}$ largest country in the world and the $4^{\text {th }}$ in Eurasia and has a population of 17 million. The country is administratively divided into 14 regions (oblasts), each of which subdivided into administrative districts (rayons). In total, there are 179 rayons with areas ranging from 7530 to $7820 \mathrm{~km}^{2}$ in the regions of North and South Kazakhstan, but can reach $38,910 \mathrm{~km}^{2}$ in Karaganda region, which is situated in the centre of the country. In terms of population, the rayons range from 5.0 to 5.8 persons per $\mathrm{km}^{2}$ in Akmola region, situated just north of Karaganda, to 23.7 persons per $\mathrm{km}^{2}$ in the region of South Kazakhstan (Figure 1). Livestock farming, especially cattle breeding, is one of the priority sectors of RK's economy. As of 1 January 2015, the number of (thousand heads) livestock cattle reached 6028.7, small cattle - 17,911.3 (sheep - 15,532.4, goats - 2378.9), pigs - 844.2, horses - 1936.7, camels - 165.9, birds - 35,000.7, domesticated Marals and Sika deer - 750.0.

\section{Data}

Data on the rabies outbreaks in RK from 2003 to 2014 were provided by the veterinary services of administrative territories (region and rayon) during visits. The database includes 762 registered cases of rabies in animals (Table 1). To perform modelling, the animals were

Table 1. List of animals registered as infected by rabies in the 2003-2014 period.

\begin{tabular}{lc} 
Species & Infected animals (n) \\
Camel & 11 \\
Cat & 13 \\
\hline Cow & 342 \\
Dog & 157 \\
\hline Fox & 166 \\
Horse & 28 \\
\hline Sheep & 38 \\
Wolf & 7 \\
\hline
\end{tabular}

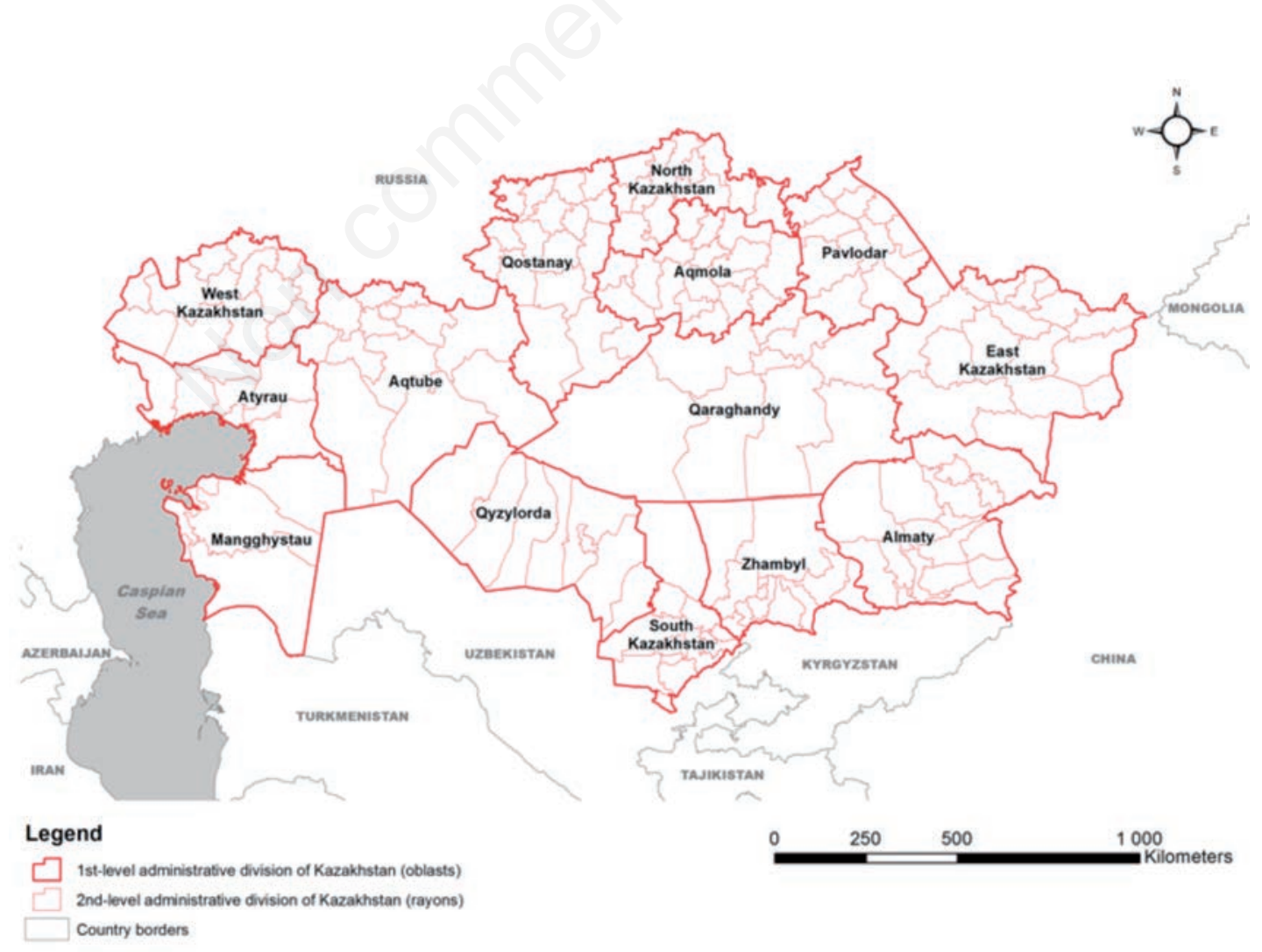

Figure 1. Administrative map of Kazakhstan. 
divided into three categories with cats and dogs counted as domestic animals (170 cases), wolves and foxes as wild animals (173), and horses, cows, sheep and camels as farm livestock (419). Figure 2 shows an administrative map of RK overlaid with cases of rabies in these three categories.

The database contains the following records on each outbreak, which are relevant to the further modelling: geographic coordinates (latitude and longitude); date of outbreak; number and type of infected animals; name of the rural settlement, rayon and region where infection occurred. All data were converted into ESRI shape-file format for the purpose of cartographic representation.

The following factors were used as explanatory variables.

First, the BI01-19 set of bioclimatic variables (bioclim), derived from the remotely sensed data on temperature and precipitation on the Earth's surface (Appendix). Data are available on the worldclim.org website (WorldClim, 2015). We used the current data set for 1950-2000.

Second, data on altitude above the mean sea level (ALT) in meters (WorldClim, 2015).

Third, data on the maximum green vegetation fraction, reflecting the presence and intensity of vegetation cover. The average for 20012012 was used [United States Geological Survey (USGS, 2015)].

Forth, land cover data for 2001-2010 (USGS, 2015). Categories of land cover are specified in Appendix.

All the variables were clipped by the contour of RK, resampled to a common spatial resolution of $1 \mathrm{xl} \mathrm{km}$ and converted into the ASCII format, which is required for modelling in MaxEnt software package.

\section{Software}

Cartographic preparation and processing as well as visualisation of data were made using geographical information system (GIS) ArcGIS, version 10.3.1 (ESRI, Redlands, CA, USA) (ESRI, 2015). The MaxEnt software package (Princeton University, Princeton, NJ, USA; http://www.cs.princeton.edu/ schapire/maxent/) was used for modelling based on the method of maximum entropy.

\section{Methodology}

To identify the predominant trend of rabies outbreaks in animals in areas with a specific combination of natural and climatic conditions we used the modelling by the maximum entropy method (Phillips et al., 2006; Elith et al., 2011). The principle is geospatial regression establishing a relationship between precisely known locations of the phenomenon under study (presence data) and a set of the potential risk factors (tipically geographical, climatic, socio-economic and miscellaneous) in the territory under study. The essence of the maximum entropy method is to obtain a probability distribution that most closely describes the known pattern of the phenomenon under study, i.e. has the maximum information entropy. The advantage of this method is that it requires presence data only, which are easily available in many

Table 2. Risk categorisation and zoning.

\begin{tabular}{lcc} 
Probability value (\%) & Risk level & Type of zone \\
$<10$ & Negligible & Disease-free (favourable) \\
$10-25$ & Low & Surveillance \\
\hline $25-50$ & Medium & Vaccination \\
$>50$ & High & Unfavourable \\
\hline
\end{tabular}
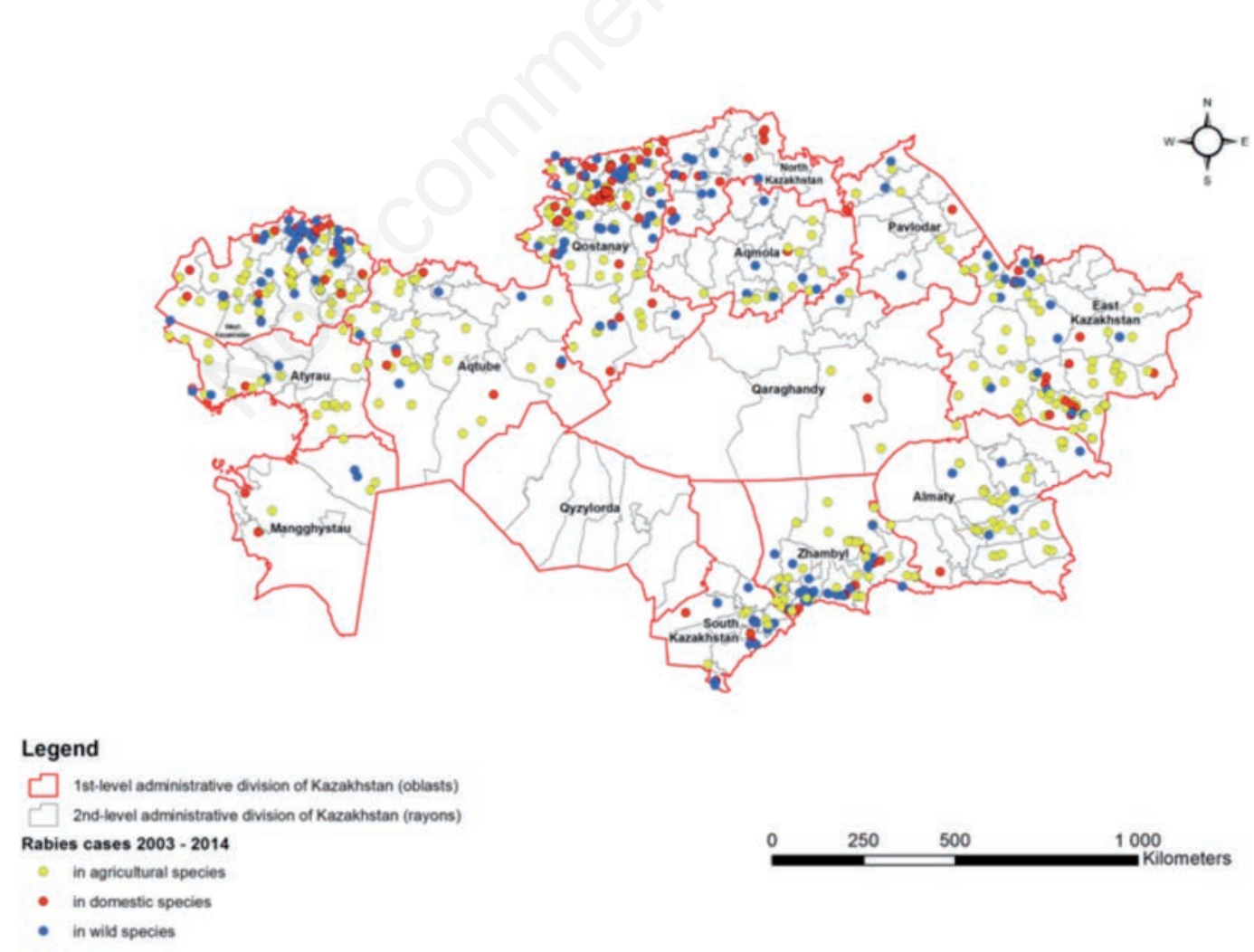

Figure 2. Administrative map of Kazakhstan and rabies cases in three categories of animals in the period 2003-2014. 
A

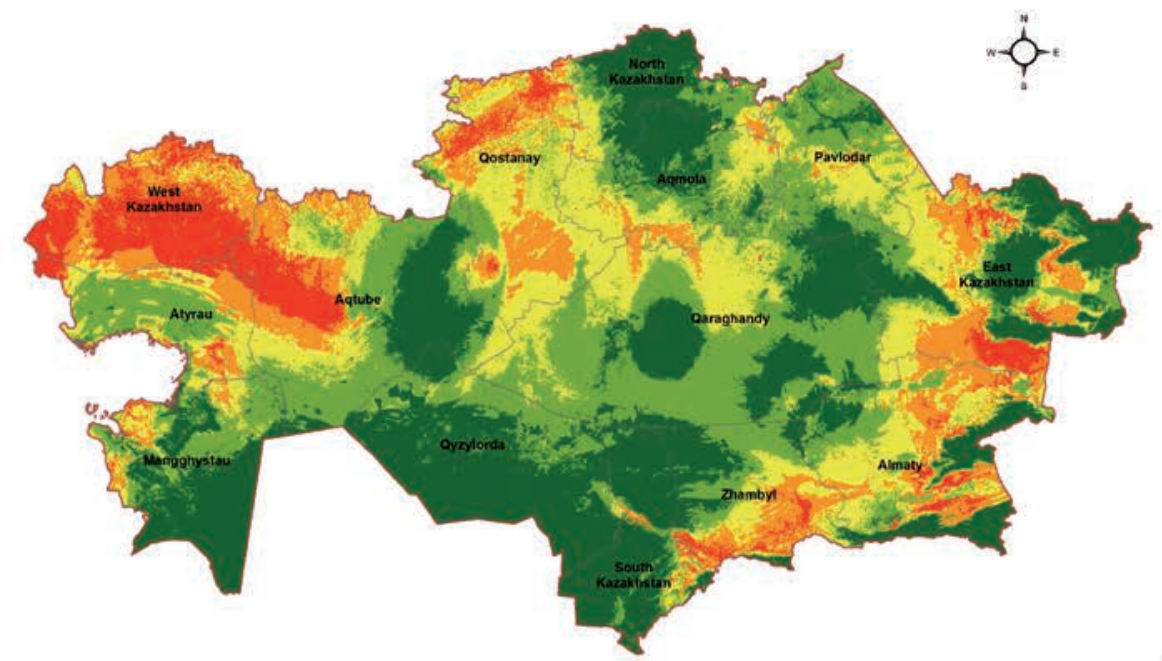

\section{Legend}

1st-tevel administrative division of Kazakhstan suitability for rabies in agricultural species less than $11 \%$

$11 \% .25 \%$

$\square 26 \%-40 \%$

$41 \%-59 \%$
more than $60 \%$

B

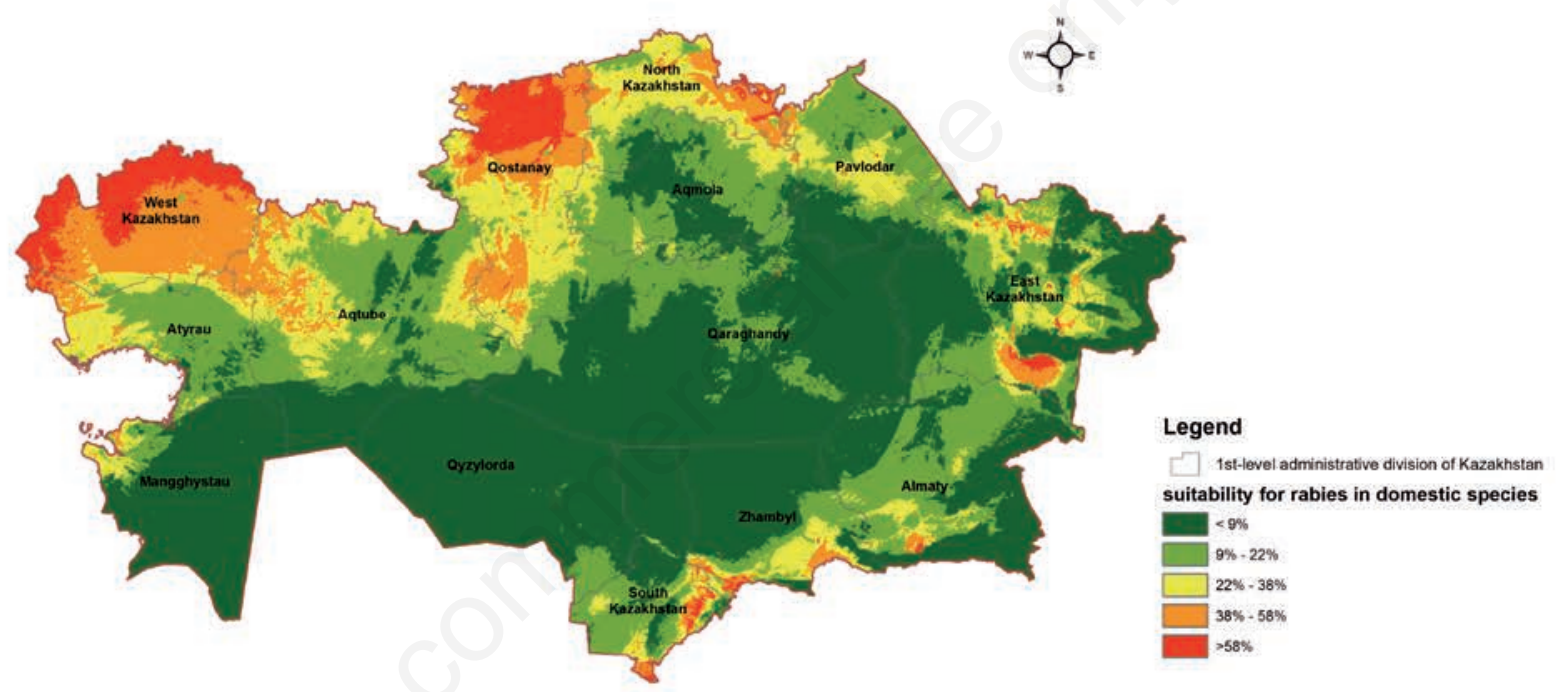

C

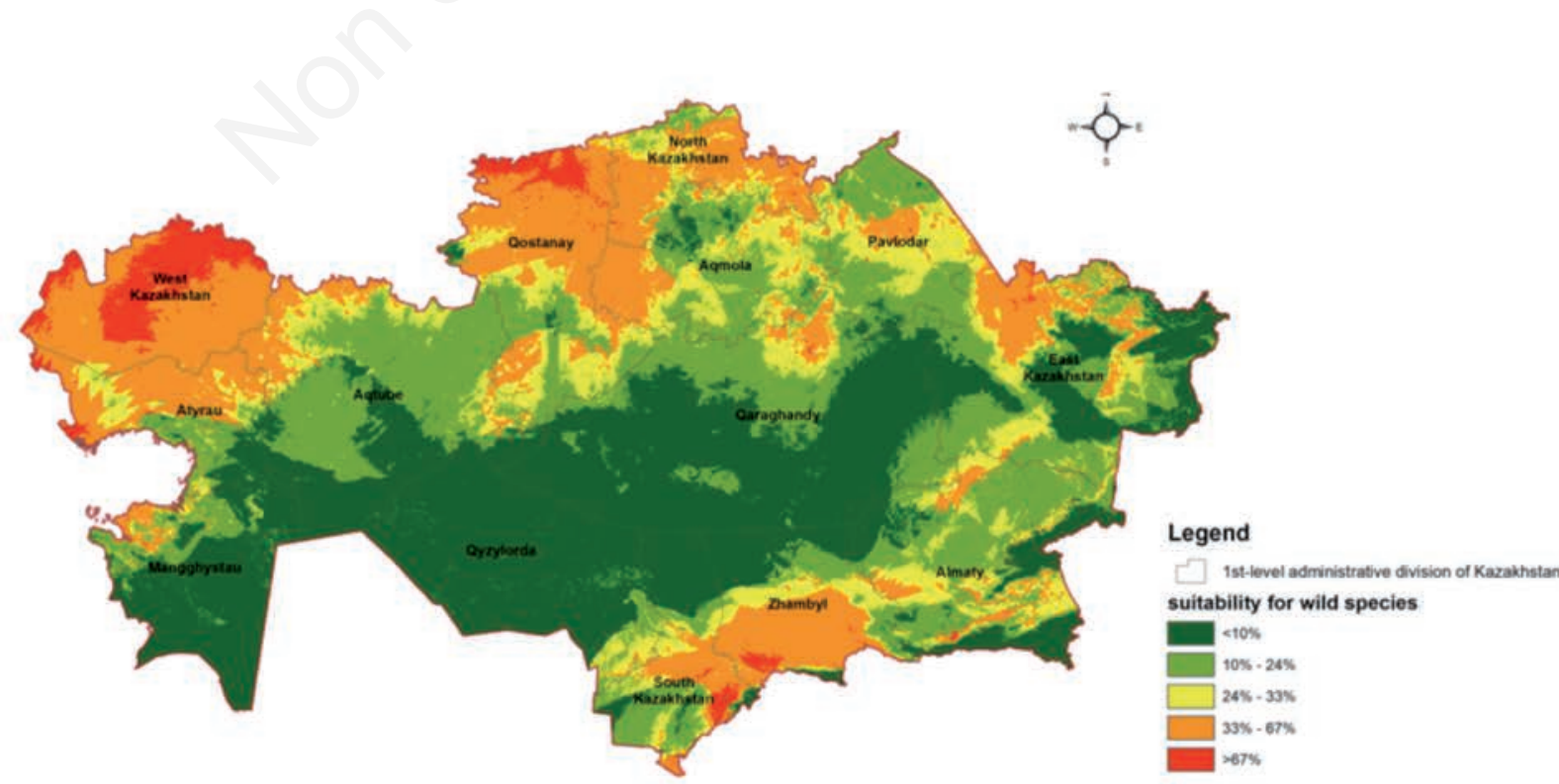

Figure 3. The probability distribution of outbreaks in farm (A), domestic (B), and wild (C) animals. 
cases. This method is typically applied to simulate the habitat of certain species based on i) precisely known locations where specimens of the species can be found, and ii) a set of explaining environmental variables within the territory (Phillips et al., 2006; Illoldi-Rangel et al., 2012; Pedersen et al., 2014). However, some studies apply the maximum entropy method to simulate the suitability of an area to the emergence of a particular disease's cases (Stevens and Pfeiffer, 2011; Mischler et al., 2012; Korennoy et al., 2014). In this case, the variables describing the socio-economic conditions in the study area can be used along with environmental variables. In our study, we also used the maximum entropy method to identify areas at risk of animal rabies outbreaks. Recorded locations of rabies cases were used as presence data.

Modelling by MaxEnt was performed separately for each of the three categories of animals: domestic, wild animals and farm livestock. The modelling of each category was performed in 10 iterations using crossvalidation to obtain average values and confidence intervals. This means that in each iteration all input data were randomly split into equal number of folds and each fold in turn was excluded from modelling, an approach which allows using all available data for validation.

To compensate for the possible bias of data caused by uneven diagnostics near populated areas, the road density grid for RK was used. This expresses the assumption that cases are more likely to be diagnosed in a close proximity to settlements and roads. To build the road density grid, we used data on highways, available in the ESRI global database of 2013 (ESRI, 2015). The density grid was built using the Kernel Density procedure from ArcGIS geoprocessing toolbox with the same spatial resolution (1x1 km).

After obtaining continuous risk surfaces with MaxEnt, they were generalised by the boundaries of administrative rayons by calculating the average value of the risk through the territory of each rayon. Risk values were categorised according to the scale presented in Table 2.

During the final stage, the three maps that represent risk to the farm livestock, domestic and wild animals were combined into an integral risk map. Thus, the largest of the three values in each category was considered as the integral value of the risk for each rayon.

\section{Results}

Distributions of the mean probabilities of outbreaks (the suitability surfaces) were obtained for each of the three categories of animal, converted into shape-files and presented in maps format (Figure 3A-C).

Receiver operating characteristic (ROC) curves, which reflect the ability of the model to explain the data available, are presented in Figure 4A-C. Here and below the red line of the graph shows the mean values and the blue field shows boundaries of $95 \%$ confidence interval obtained by multiple model runs. The predictive accuracy of the model, expressed as area under the ROC curve, i.e. the area under the curve (AUC) value, is: $0.782 \pm 0.031$ for the farm livestock category; $0.859 \pm 0.042$ for the domestic animals and $0.809 \pm 0.045$ for the wild animals. It is usually considered that AUC values around 0.5 have no predictive power, while AUC values $>0.7$ are acceptable and those above 0.8 sufficiently high to indicate a strong ability of the model to explain available data (Elith et al., 2011). Thus, the probability distributions obtained allowed us, with a high degree of reliability, to describe the distribution of existing rabies cases in RK as depending on the set of prevailing climatic and geographical factors in the different locations investigated. It was decided to emphasise those variables that contributed $10 \%$ and more to the modelling results and present them as the important ones.
The response curves are shown in Figure 5. For the farm livestock category (Figure 5A), the variables that contributed most to the model were BI019, LANDCOV and BI01, for the domestic animals category, they were LANDCOV, ALT, BIO12 and BIO19 (Figure 5B) and for the wild animals category, LANDCOV, BI019, ALT and BIO12 (see Appendix for explanation of the abbreviated bioclim variables).

Following the integration of risk values by the three animal categories, a final picture of regionalisation was obtained (Figure 6 ). This map shows the result of regionalisation (zoning) in RK between the four risk categories among the various species.

The probability distribution of rabies outbreaks (Figure 3A) among farm species demonstrating more than $60 \%$ risk of cases was seen in West Kazakhstan, Aktobe, Kostanay, East Kazakhstan, Almaty, Zhambyl and partially Atyrau, Mangistau and South Kazakhstan regions, while

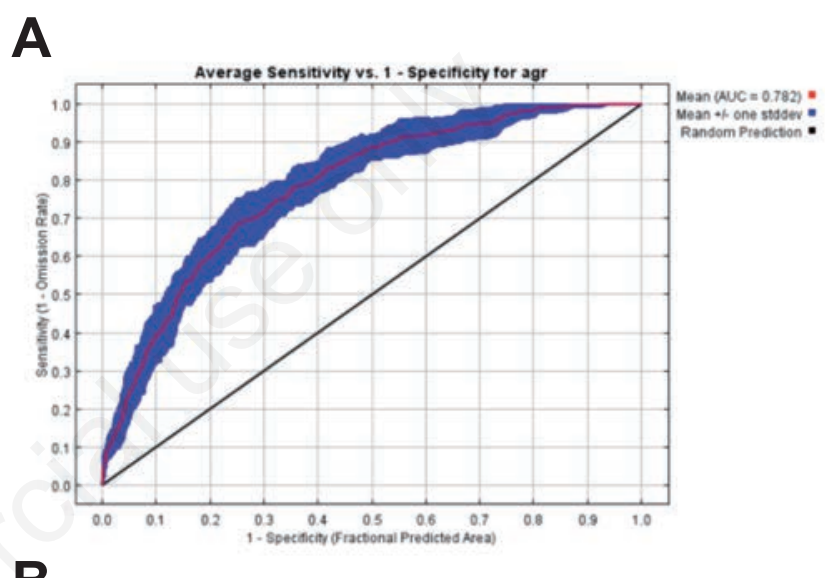

B

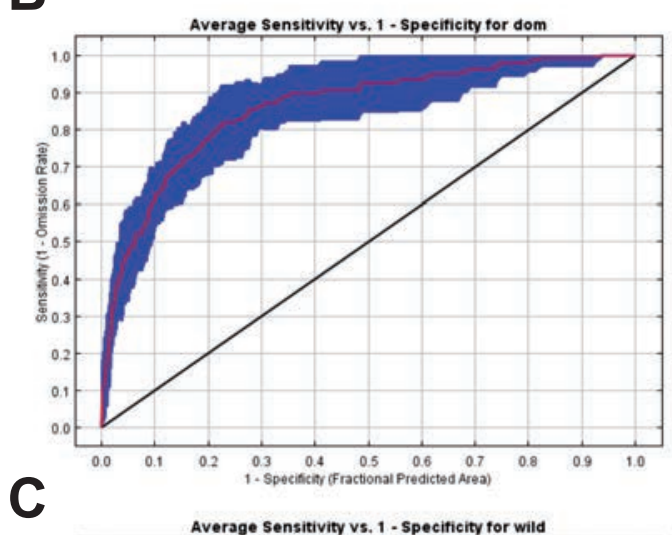
Mean (auC $=0.859$ :
Mean + one stddever :
Random Prediction :

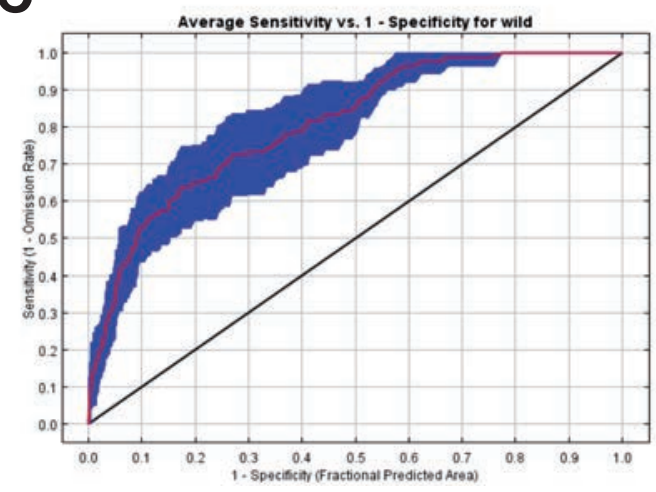

Mean $(A U C=0.809)=$ Mandom Prediction :

Figure 4. Receiver operating characteristic-curve for the category farm (A), domestic (B), and wild (C) animals. 
A
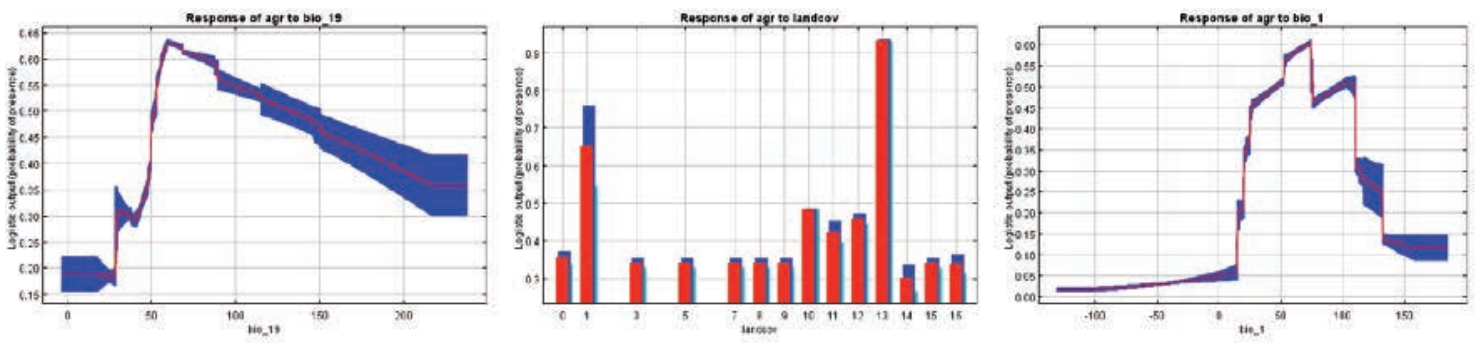

B
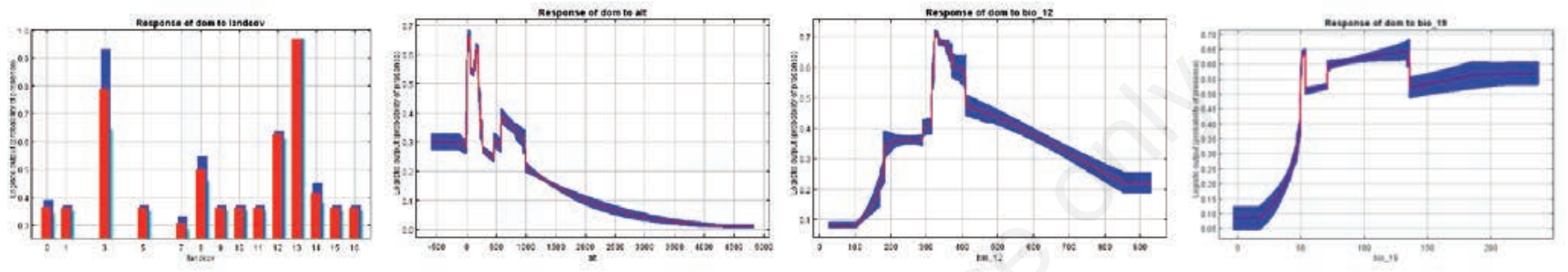

C
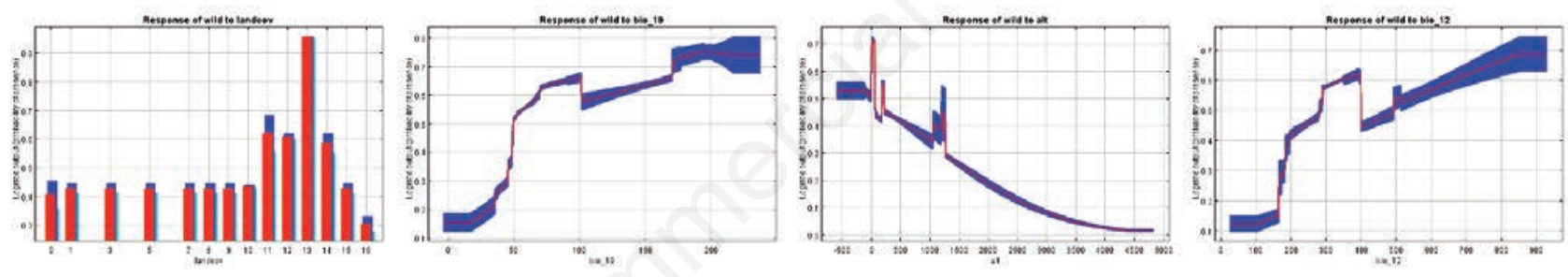

Figure 5. Response curves for the variables BIO 19, LANDCOV and BIO1 (left to right) for the model farm animals (A); variables LANDCOV, ALT, BIO12 and BIO19 (left to right) for the model domestic animals (B); and variables LANDCOV, BIO 19, ALT, and BIO12 (left to right) for the model wild animals $(\mathrm{C})$. Red colour shows the mean values and the blue field shows boundaries of $95 \%$ confidence interval obtained by multiple model runs.

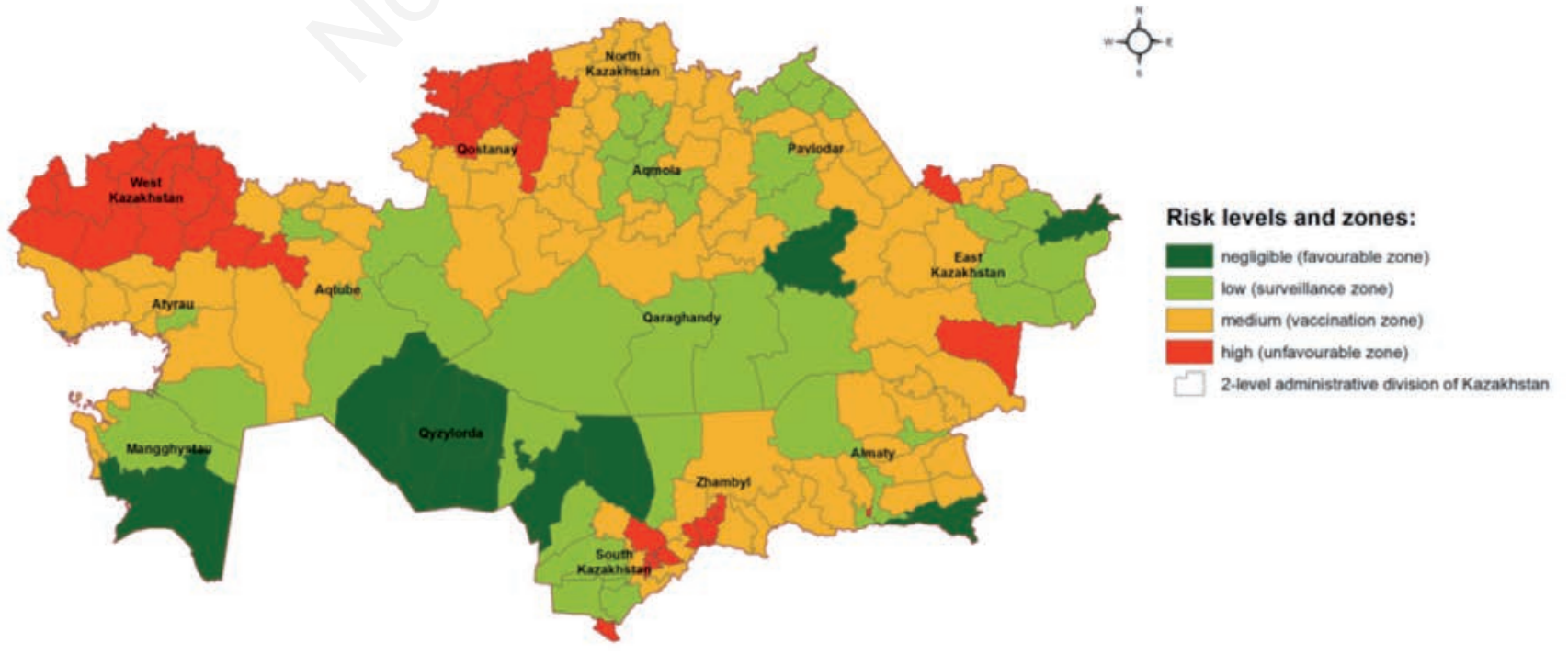

Figure 6. The integrated zoning map of the Republic of Kazakhstan in terms of animal rabies risk. 
the lowest probability (less than 11\%) was mostly observed in areas such as North Kazakhstan, Akmola, Karaganda, Kyzylorda and the largest part of Mangistau, South Kazakhstan regions, partly Aktobe, Pavlodar, East Kazakhstan, Almaty and Zhambyl regions.

With regard to the distribution of the probability of rabies occurrence in domestic animals (Figure 3B), it was noted that more than $58 \%$ of outbreaks of rabies accounted for almost half of the territory of Western Kazakhstan, one third of Kostanai regions and partly of the North Kazakhstan oblast, East Kazakhstan, South Kazakhstan, Zhambyl and Almaty regions. The lowest value (less than 9\%) was observed in most parts of the country, namely, Mangistau, Kyzylorda, and Karaganda, much of the Akmola, South Kazakhstan, Zhambyl, partly Aktobe, Atyrau, Pavlodar, East Kazakhstan and Almaty regions.

In the case of wild animals (Figure 3C), the situation was somewhat different, with the highest probability of rabies occurrence (over 67\%) observed in the West Kazakhstan and Kostanai regions, partly Atyrau, South Kazakhstan, Zhambyl and to a lesser extent the East Kazakhstan and Almaty regions. Almost a similar picture emerges as to the minimum occurrence of rabies (less than 10\%), both livestock and wild animals found throughout most of Mangistau, Kyzylorda, Karaganda regions, partly Atyrau, Aktobe, East Kazakhstan, Almaty, Zhambyl and South Kazakhstan regions, and slightly in Kostanai, North Kazakhstan, Akmola, Pavlodar regions.

\section{Discussion}

Many authors (Bersagurov, 2002; Kiryakova et al., 2004; Abdrakhmanov et al., 2010) associate the probability distribution of rabies outbreaks in the country with the influence of combined climatic and socio-economic factors. Although we did not study the social and economic influence, we also identified climatic variables as being important influencing the spread of rabies among different categories of susceptible animals (Figure 5A-C). The most important ones were the prevailing type of land cover and the amount of preciptation in the coldest quarter that were both found for all three categories, while the average annual rainfall and altitude were also important. These findings together with the territorial distribution of risk (Figure 2) support an assumption of existence of natural disease's foci and of strong interference between epizootic processes in these sub-populations. This interference is also supported by a previous correlation analysis conducted by (Abdrakhmanov et al., 2010). In this study, it was found that the correlation coefficient between the incidence of rabies in populations of wild and domestic animals over time amounts to 0.68 . This suggests one infected fox may increase the incidence of rabies in domestic carnivores and as a consequence among productive animals by 2-3 heads.

Appendix demonstrates the distribution of administrative rayons in RK by risk areas in accordance with Figure 6 . Attention should be paid to the fact that the area of highest risk is concentrated mainly at the borders of RK with neighbouring countries, i.e. the Russian Federation, Uzbekistan, Kyrgyzstan and China. This may indicate the presence of rabies importation from the territory of these states. This epidemiologically significant fact requires further science-based mathematical analysis and joint research to improve the epizootic situation. Similar results were obtained by many researchers who study the nature of the rabies spread (Shestopalov et al., 2001; Yin et al., 2013). In addition, a high density of settlements and animals (domestic and farm livestock, wild animals) was registered in the border regions of RK, where most reported cases of rabies in animals occur.
Further work on geospatial analysis of rabies epizootic in Kazakhstan may include the introduction of additional variables in the model. In particular, these variables may include distribution of populations of susceptible animals in the territory and the proximity to sources of infection in the territory of neighbouring states.

\section{Conclusions}

Based on the results obtained, guidelines have been developed to arrange the preventive and anti-epizootic measures against rabies in the regions of RK. Depending on the risk zone, the region falls into measures ranging from system of rigid veterinary-sanitary actions (including vaccination) to monitoring and verification. Guidelines have been communicated to the national authorities and are expected to become a basis for a national programme for the prevention and control of rabies.

\section{References}

Abdrakhmanov SK, Sytnic II, Tursunkulov SZh, 2010. Visualization and analysis of veterinary and geographical rabies spread by using GIS technologies. In: Proceedings of the 5th International Scientific Practical Conference, 2010 March 17-18, Barnaul. AGAU Publ., Barnaul, Russia, pp 283-6.

Bersagurov KA, 2002. Epizootic and epidemiological situation of rabies in the West. Kazakhstan region and prevention measures. Official Bulletin of the State Sanitary and Epidemiological Service of the Republic of Kazakhstan 2002:24-30.

Chubirko MI, Chervanev VA, Efanova LI, 2003. Epizootiology of rabies in Voronezh region. Veterinary and medical aspects of zooanthroponosis. Pokrov 2003:102-7.

Domsky IA, 2002. Natural foci of rabies and its principal owners. Vet Pathol 1:119-22.

Dudnikov SA, 2003. Foxes as a marker of risk in rabies: epizootological aspects. Veterinary and medical aspects of zooanthroponosis. Pokrov 2003:69-73.

Elith J, Phillips SJ, Hastie T, Dudik M, Chee YE, Yates CJ, 2011. A statistical explanation of MaxEnt for ecologists. Diversity and Distributions 17:43-57.

ESRI, 2015. GIS mapping software, solutions, map series, apps and data. Available from: http://www.esri.com/

Illoldi-Rangel P, Rivaldi C-L, Sissel B, Fryxell RT, Gordillo-Perez G, Rodriguez-Moreno A, Williamson P, Montiel-Parra G, SanchezCordero V, Sarkar S, 2012. Species distribution models and ecological suitability analysis for potential tick vectors of lyme disease in Mexico. J Trop Med 2012:959101.

Kiryakova LS, Khaytova AB, Kovalenko IS, 2004. The use of geographical information technologies in the epidemiological diagnosis of highly dangerous infections. Probl Dang Inf 87:24-7.

Korennoy FI, Gulenkin VM, Malone JB, Mores CN, Dudnikov SA, Stevenson MA, 2014. Spatio-temporal modeling of the African swine fever epidemic in the Russian Federation, 2007-2012. Spatial and Spatiotemporal Epidemiol 11:135-41.

Makarov VV, Sukhareva OI, Gulyukin AM, Litvinov OB, 2008. The trend of the rabies spread in Eastern Europe. Vet Med 7:20-2.

Mischler P, Kearney M, McCarroll JC, Scholte RGC, Vounatsou P, Malone JB, 2012. Environmental and socio-economic risk model- 
ling for Chagas disease in Bolivia. Geospat Health 6:59-66.

Nouvellet P, Donnelly CA, De Nardi M, Rhodes CJ, De Benedictis P, Citterio C, Obber F, Lorenzetto M, Pozza MD, Cauchemez S, Cattoli G, 2013. Rabies and canine distemper virus epidemics in the red fox population of northern Italy (2006-2010). PLoS One 8:e61588.

Orłowska A, Smreczak M, Trębas P, Żmudziński JF, 2011. Rabies outbreak in Małopolska region in Poland in 2010. Bull Vet Inst Pulawy 55:555-61.

Pedersen UB, Midzi N, Mduluza T, Soko W, Stensgaard A-S, Vennervald BJ, Mukaratirwa S, Kristensen TK, 2014. Modeling spatial distribution of snails transmitting parasitic worms with importance to human and animal health and analysis of distributional changes in relation to climate. Geospat Health 8:335-43.

Phillips SJ, Anderson RP, Schapire RE, 2006. Maximum entropy modeling of species geographic distributions. Ecol Model 190:231-59.

Robardet E, Ilieva D, Iliev E, Gagnev E, Picard-Meyer E, Cliquet F, 2013. Epidemiology and molecular diversity of rabies viruses in Bulgaria. Epidemiol Infect 5:1-7.

Shestopalov AM, Kissurina MI, Gruzdev KN, 2001. Rabies and its distribution in the world. Probl Virol 2:7-12.

Smreczak M, Orłowska A, Trębas P, Żmudziński JF, 2012. Rabies epidemiological situation in Poland in 2009 and 2010. Bull Vet Inst
Pulawy 56:115-266.

Smreczak M, Orłowska A, Żmudziński JF, 2009. Rabies situation in Poland in 2008. Bull Vet Inst Pulawy 53:583-7.

Stevens KB, Pfeiffer DU, 2011. Spatial modeling of disease using dataand knowledge-driven approaches. Spatial Spatiotemporal Epidemiol 2:125-33.

USGS, 2015. United States Geological Survey Land Cover Institute. Available from: http://andcover.usgs.gov/

WorldClim, 2015. Global climate data. Available from: http://worldclim.org/

Yin W, Dong J, Tu C, Edwards J, Guo F, Zhou H, Yu H, Vong S, 2013. Challenges and needs for China to eliminate rabies. Infect Dis Poverty 2:23.

Youla AS, Traore FA, Sako FB, Feda RM, Emeric MA, 2014. Canine and human rabies in Conakry: epidemiology and preventive aspects. Bull Soc Pathol Exot 7:19-21.

Zavodskih AI, Sludov AI, 2007. The behaviour of raccoon dogs infected with the rabies. Vet Med 2:15-6.

Zholshorinov AZ, Sansyzbayev YB, 2004. Surveillance of rabies in wildtype centres of domination: the methodical recommendations. Astana 2004:16. 